\title{
Old Immunosuppressive Agents: Newer Approach in liver Transplant Recipients to Achieve Rejection Free and Nephron Preserving Protocol
}

\section{Ashok Kumar Jain* and Amar Nath Mukerji}

Department of Transplantation, Temple University Hospital, Philadelphia, PA, USA

\begin{abstract}
In liver transplant (LTx) recipient bioavailability of oral Mycophenolate Mofetil (MMF) is less than $48 \%$ in first weeks post LTx week. Intravenous (IV) MMF allows $100 \%$ bioavailability with higher peak values. Use of MMF in immediate post LTX period allows delayed introduction of tacrolimus which allows preserving the renal function and reversing the pre-existing renal dysfunction promptly after successful LTx without use of antibody preparation and at no risk of precipitating acute cellular rejection.
\end{abstract}

Keywords: Liver transplant; Acute rejection; Intravenous MMF; Renal function

Combination of Calcineurine Inhibitor $(\mathrm{CN})$, antiprolifeative agent and steroid has become the standard of immunosuppressive strategy in most liver transplant (LTx) centers in immediate post-operative period. Among stantiproliferative agents mycophenolatemofetil (MMF) is most commonly used in majority of transplant centers is the U.S. Incidence of acute cellular rejection (ACR) is much lower in the current era compared to the past. However, ACR does occur and majority of them in first few weeks after LTx [1]. Also, as more sick patients are being transplanted, organs are first offered to the sickest patients with high MELD score. Patients with higher MELD scores invariably have a degree of renal dysfunction or even renal failure requiring renal replacement therapy mainly for hepatorenal syndrome type I or type II. Also, up to $20 \%$ of patients who were not on dialysis pre-transplant may require dialysis in post-transplant period $[2,3]$. Various immunosuppressive approaches have been cited with use of antibody preparation in group of patient with pre-existing renal dysfunction including IL-2 inhibitors and Thymoglobulin [4]. We have in the past published the use of intravenous MMF instead of oral MMF in immediate post-operative period without antibody induction, both to reduce the rate of ACR and preserve renal function [5-7]. The rationale for I.V. use of MMF versus oral is based on kinetic studies performed in post-LTx patients, which is different from healthy volunteers and other solid organ transplant recipients. It was reported that more than $90 \%$ of oral MMF is absorbed in healthy volunteers, renal, heart and lung transplant patients. Whereas in liver transplant patients only $48 \%$ of the drug is absorbed in second week after LTx which could be even lower in the first week after LTx based on rising trough level of MMF in first month and also rising bioavailability of MMF in $1^{\text {st }}$ week to $2^{\text {nd }}$ week to $4^{\text {th }}$ week post-LTx [8-11].

Aim of the present study is to examine the benefit of I.V. MMF in Perioperative period following LTx with lower dosage and progressively delayed use of oral tacrolimus in providing rejection free and renal preservation immunosuppressive protocol in a carefully monitored approach.

The efficacy of I.V. MMF was first published in 2005; where 28 consecutive live donor LTx patients received I.V. MMF with delayed use of oral tacrolimus for up to 24 hours. Two patients (7.1\%) experienced ACR (rejection activity index, RAI 2-3) within the first year post-LTx. Both ACR resolved with methylprednisolone bolus without antibody therapy. One patient (3.4\%) required dialysis for 9 days [5].

Another study was published with a larger population (2008) consisting of 112 deceased donor and 18 living donor LTx during year 2005 with mean follow-up of 12 months. Nine patients (6.9\%) experienced ACR (RAI >3) requiring methylprednisolone bolus to treat without antibody preparation. Seven of them had sub-therapeutic tacrolimus levels and were known to be non-compliant in taking immunosuppressant medications [6].

In our current experience of 72 consecutive LTxs in a new program, all received I.V. MMF twice daily; first dose before reperfusion and introduction of oral tacrolimus was delayed from 2 to 6 days in 48 patients. Of these 48 patients in 31 the tacrolimus was delayed for 2 to 4 days and none of them experienced ACR. In the remaining 17 patients, tacrolimus was introduced on post-operative day $5(n=14)$ and post-operative day $6(n=3)$. Of these 17 patients, one patient experienced ACR on post-operative day $8(\mathrm{RAI}=7)$. He required total 2 gm methylprednisolone over 5 days. Rejection was completely resolved without use of antibody preparation. Another patient who was noncompliant in taking her medications experienced ACR 4 month's postLTx which was resolved with $1.5 \mathrm{gm}$ of methylprednisolone.

More interestingly, 9 patients were on hemodialysis before transplant for $1-14$ weeks. All of them came off dialysis. Two patients expired, one form cardiopulmonary arrest and another from metastatic hepatocellular carcinoma. The remaining 7 patients are off renal replacement therapy for 28 to 45 months.

Two patients, who required short term dialysis after LTx, were not on dialysis pre-LTx. Both had complicated post-operative course; one of them had undergone re-transplantation for primary non-function.

We are convinced that I.V. MMF (instead of oral MMF) and delayed use of tacrolimus with standard use of steroid provides rejection free survival in up to $97 \%$ of cases. Delayed introduction of calcineurin inhibitors provides the renal protection and allows early recovery from pre-transplant dialysis need and also from the need of new onset dialysis post-LTx.

*Corresponding author: Ashok Kumar Jain, Department of Transplantation Temple University Hospital, Philadelphia, PA-19140, USA, Tel: +1-215-707-8538; Fax: +1-215-707-5960; E-mail: Ashokkumar.Jain@tuhs.temple.edu

Received January 19, 2013; Accepted April 28, 2013; Published May 07, 2013

Citation: Jain AK, Mukerji AN (2013) Old Immunosuppressive Agents: Newer Approach in liver Transplant Recipients to Achieve Rejection Free and Nephron Preserving Protocol. Surgery S12: 014. doi:10.4172/2161-1076.S12-014

Copyright: (๑) 2013 Jain AK, et al. This is an open-access article distributed under the terms of the Creative Commons Attribution License, which permits unrestricted use, distribution, and reproduction in any medium, provided the original author and source are credited. 
Citation: Jain AK, Mukerji AN (2013) Old Immunosuppressive Agents: Newer Approach in liver Transplant Recipients to Achieve Rejection Free and Nephron Preserving Protocol. Surgery S12: 014. doi:10.4172/2161-1076.S12-014

\section{References}

1. Eckhoff DE, McGuire BM, Frenette LR, Contreras JL, Hudson SL, et al. (1998) Tacrolimus (FK506) and mycophenolate mofetil combination therapy versus tacrolimus in adult liver transplantation. Transplantation 65: 180-187.

2. Pawarode A, Fine DM, Thuluvath PJ (2003) Independent risk factors and natural history of renal dysfunction in liver transplant recipients. Liver Transpl 9: 741-747.

3. Wong LP, Blackley MP, Andreoni KA, Chin H, Falk RJ, et al. (2005) Survival of liver transplant candidates with acute renal failure receiving renal replacement therapy. Kidney Int 68: 362-370.

4. Tchervenkov JI, Tzimas GN, Cantarovich M, Barkun JS, Metrakos P (2004) The impact of thymoglobulin on renal function and calcineurin inhibitor initiation in recipients of orthotopic liver transplant: a retrospective analysis of 298 consecutive patients. Transplant Proc 36: 1747-1752.

5. Jain A, Mohanka R, Orloff M, Abt P, Kashyap R, et al. (2005) Intravenous mycophenolate mofetil with low-dose oral tacrolimus and steroid induction for live donor liver transplantation. Exp Clin Transplant 3: 361-365.

6. Jain A, Sharma R, Ryan C, Tsoulfas G, Orloff M, et al. (2005) Potentia immunological advantage of intravenous mycophenolate mofetil with tacrolimus and steroids in primary deceased donor liver transplantation and live donor live transplantation without antibody induction, Liver Transpl 14: 202-209.

7. Jain A, Vekatramanan R, Eghtesad B, Gadomski M, Mohanka R, et al (2005) Long-term outcome of adding mycophenolate mofetil to tacrolimus for nephrotoxicity following liver transplantation. Transplantation 80: 859-864.

8. Jain A, Venkataramanan R, Hamad IS, Zuckerman S, Zhang S, et al (2001) Pharmacokinetics of mycophenolic acid after mycophenolate mofetil administration in liver transplant patients treated with tacrolimus. J Clin Pharmacol 41: 268-276.

9. Jain A, Venkataramanan R, Kwong T, Mohanka R, Orloff M, et al. (2007) Pharmacokinetics of mycophenolic acid in liver transplant patients afte intravenous and oral administration of mycophenolate mofetil. Liver transpl 13 791-796

10. Jain A, Venkataramanan R, Sharma R, Kwong T, Abt P, et al. (2008) Pharmacokinetics of mycophenolic acid in live donor liver transplant patients vs deceased donor liver transplant patients. J Clin Pharmacol 48: 547-552.

11. Pisupati J, Jain A, Burckart G, Hamad I, Zuckerman S, et al. (2005) Intraindividual and interindividual variations in the pharmacokinetics of mycophenolic acid in liver transplant patients. J Clin Pharmacol 45: 34-41. 